TUBB3

$4: 00 p m-4: 15 p m$

\title{
Wavelength Dependence of Catastrophic Optical Damage Threshold in 980nm Semiconductor Diode Lasers
}

\author{
Daren Lock, Stephen J. Sweeney and Alfred R. Adams

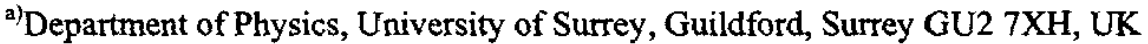 \\ +44 (0) 1483 689406, Fax: +44 (0) 1483689404
}

\begin{abstract}
We investigate the wavelength dependence of the catastrophic optical damage current in 980nm lasers. Using high pressure and low temperature techniques, we find an intrinsic dependence of this threshold on wavelength.
\end{abstract}

\section{Summary}

There is a growing demand for high power semiconductor lasers with applications ranging from domestic use in recordable storage formats such as digital versatile discs (DVD-RW) and compact discs (CD-RW), for printing and marking technologies, for telecommunications systems and increasingly in medical applications such as field surgery and phototherapy. Telecommunications, in particular, the development of Erbium-Doped Fiber Amplifiers (EDFAs) gave rise to the need for semiconductor lasers emitting several hundred milliwatts of power at $980 \mathrm{~nm}$ or $1480 \mathrm{~nm}$. More recently, thete has been widespread interest in developing distributed Raman amplifiers which require semiconductor lasers emitting in the range $\sim 1430-1460 \mathrm{~nm}$ (for C-band amplification) with fiber coupled powers $\sim 0.5 \mathrm{~W}$. Thus, for many applications, output powers of several hundred milliwatts are required frequently giving rise to power densities at the laser facets $\sim 1-10 \mathrm{MWcm}^{-2}$. Such high power densities can result in damage of the facet itself, where strong re-absorption of the emitted radiation causes extreme heating. This leads to band gap shrinkage close to the facet, giving rise to further absorption in a self-depreciating manner. At the extreme this causes the facet to melt and a catastrophic drop in output power occurs. Absorption at the laser facet can be due to both internal (layer composition, strain') and external properties of the laser (facet coatings, local atmosphere). $\mathrm{COD}$ is known to be a significant problem in $\mathrm{Al}(\mathrm{GaInP} / \mathrm{GaAs}$ visible lasers and $\mathrm{InGaAs} / \mathrm{GaAs}$ devices at $980 \mathrm{~nm}$. However, InP-based devices such as those emitting at the telecommunications wavelengths $(\sim 1.3 \mu \mathrm{m}$ and $\sim 1.5 \mu \mathrm{m})$ seem immune to COD. Such devices are based upon InP, rather than GaAs as used for the shorter wavelength devices. The increased thermal conductivity of $\mathrm{InP}\left(0.68 \mathrm{WK}^{-1} \mathrm{~cm}^{-1}\right)$ compared with $\mathrm{GaAs}\left(0.44 \mathrm{WK}^{-1} \mathrm{~cm}^{-1}\right)$ may make heat dissipation more effective in GaAs. In addition, the surface recombination velocity of InP is two orders of magnitude lower than that of $\mathrm{GaAs}$ and hence surface defect related current paths are likely to be considerably more important in GaAs. Long wavelength InP-based devices also tend to suffer from overall device heating due to intrinsic inefficiencies as a result of dominant non-radiative loss processes. However it is not clear which of these mechanisms is primarily responsible for COD.

In this paper we investigate these processes in order to obtain a better understanding of which process(es) influence if and when COD occurs. Measurements were made on GaAs/AlGaAs $980 \mathrm{~nm}$ ridge waveguide lasers and $1480 \mathrm{~nm}$ InGaAs/InP buried heterostructure lasers. At room temperature (figure 1) the $980 \mathrm{~nm}$ devices were driven with pulsed current until the device underwent COD at an output power density of $\sim 10 \mathrm{MW} / \mathrm{cm}^{2}$. In comparison, the $1480 \mathrm{~nm}$ device was driven until joule heating caused the output power to rollover even under pulsed conditions. Dominant non-radiative current may stop COD occurring in longer wavelength devices as the output power simply can not reach sufficiently high power densities to undergo COD.

To test this hypothesis the devices were cooled down to $77 \mathrm{~K}$ using a nitrogen static exchange gas cryostat. At this temperature, the $1480 \mathrm{~nm}$ devices behave ideally since at this temperature non-radiative processes are negligible. It can be seen from figure 1 that the $980 \mathrm{~nm}$ device undergoes COD, at the same output power density of $\sim 10 \mathrm{MW} / \mathrm{cm}^{2}$ as at RT. The $1480 \mathrm{~nm}$ device, in the absence of loss processes, is driven over one thousand times its threshold current and reaches a power density of $\sim 18 \mathrm{MW} / \mathrm{cm}^{2}$ without 
undergoing COD. It is clear therefore that there is a major difference between the two wavelengths in terms of their susceptibility to COD
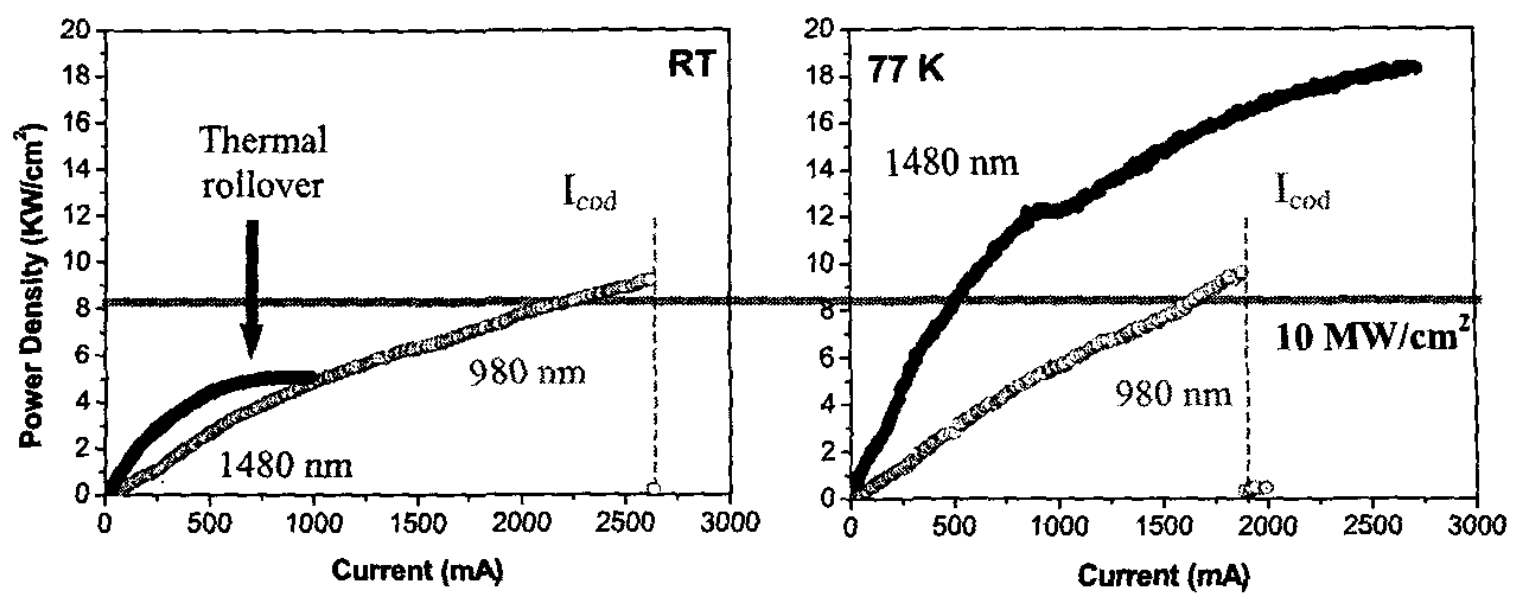

Figure 1,Facet output as a function of current for both $980 \mathrm{~nm}$ (red open circles) and $1480 \mathrm{~nm}$ (blue closed circles). The thermal rollover can be seen for the $1480 \mathrm{~nm}$ RT graph. Also marked are the currents at which the $980 \mathrm{~nm}$ devices undergo $\mathrm{COD}$

To investigate this wavelength difference we looked at the current at which the devices undergo $\mathrm{COD}, \mathrm{I}_{\mathrm{cod}}$ as a function of hydrostatic pressure and temperature (figure 2). Hydrostatic pressure enables the lasing wavelength of the device to change due to the increase in the bandgap of the material with increasing pressure. Using this technique, we can look at a range of wavelengths from devices with identical material parameters. A series of nominally identical $980 \mathrm{~nm}$ devices were driven COD under a range of pressures. We also drove a series of nominally identical $980 \mathrm{~nm}$ devices $\mathrm{COD}$ as a function of temperature in the nitrogen static exchange gas cryostat. Temperature varies the emission wavelength in accordance with the Varshni equation ${ }^{2}$.

We can see from figure 2 that $\mathrm{I}_{\mathrm{cod}}$ decreases with decreasing wavelength, both as a function of pressure and wavelength, with the same decrease observed for both sets of experiments. These results clearly show an intrinsic wavelength dependence of $I_{\text {cod. }}$ As

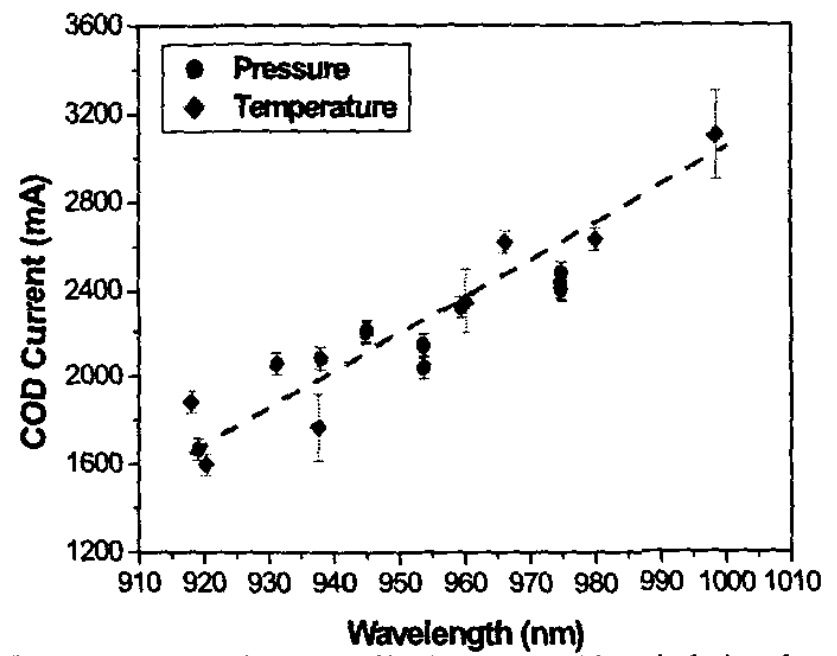

Figure 2, $I_{\text {cod }}$ as a function of both pressure (blue circles) and temperature (red diamonds) plotted against wavelength the use of pressure and temperature

eliminates extrinsic effects from the possible causes of the COD processes, we can attribute this decrease to the change in photon energy. The results therefore indicate a fundamental wavelength dependence of catastrophic optical damage. Further work into the underlying photon energy dependence of COD will be discussed.

\footnotetext{
'A. Valster et al, IEEE Jour. Sel. Top. Quant. Elect., 3, p180 (1997)

2 eproperties of Gallium Arsenide", INSPEC, 1990.
} 